\title{
CLASSES OF TRANSFORMATIONS AND BORDERING TRANSFORMATIONS
}

\author{
D. G. BOURGIN
}

Introduction. I do not propose to give a complete or closed theory of any of the phases of the subjects I touch on in this address. In fact one of my primary purposes is to indicate what the natural unsolved problems are and what it would be reasonable to expect, and I shall raise as many questions as I answer. Many of my comments bear on results not yet published or on work in progress in connection with a book on fixed point theory. In a general way the first part of my remarks will interpret bordering transformations as those metrically near a canonical type, usually an isometry, while my later remarks will interpret bordering in a homotopy sense.

Our initial considerations will be devoted to the implications of what we may term uniform departures from standard transformations. The first to formulate some problems of this type was Ulam. In order to bring out their interconnections we present them as special cases or modifications of a general form and free the formulation from metric dependence although the metric cases alone have been treated. Suppose then that $X$ and $Y$ are uniform spaces with the vicinities of the diagonals denoted by $\mathfrak{R}$ and $\mathfrak{M}$. We assume the existence of a multiplication in these spaces denoted by $O$ and $\oplus$. That is to say, $\bigcirc$ maps $X \times X$ into $X$, and $\oplus$ maps $Y \times Y$ into $Y$. Let $T, S$ be transformations on $X$ to $Y$ satisfying

$$
\left(S\left(x_{1} \bigcirc x_{2}\right), T_{x_{1}} \oplus T_{x_{2}}\right) \subset \mathfrak{M} .
$$

We assume also a class of standard transformations from $X$ to $Y$ which we denote by $U$. We may then formulate a significant problem in the following way: Does there exist a standard transformation $U$ and a vicinity $\mathfrak{R}^{\prime}$ dependent on $\mathfrak{M}$ alone such that

$$
(T x, U x) \subset \mathfrak{N}^{\prime},
$$

and what is the "best" $\mathfrak{\Re}$ '?

1. Approximate additivity and isometry. As illustration we can take for $X$ a Banach space in its norm topology and for $Y$ the real line. Let $S=T$ and let $\bigcirc$ and $\oplus$ be the respective vector addition

An address delivered before the Evanston meeting of the Society on November 24, 1950 by invitation of the Committee to Select Hour Speakers for Western Sectional Meetings; received by the editors January 27, 1951. 
operations. Here $U$ is additive and $\mathfrak{M}$ is the $\epsilon$ sphere. In more familiar guise then we rewrite (1) as

$$
\|T(x+y)-T x-T y\|<\epsilon,
$$

and ask if an additive transformation $U$ exists with

$$
\|T x-U x\| \leqq k \epsilon,
$$

where $k$ is a fixed constant for the space. Later references to $k$ invariably imply $k$ satisfies (4). This problem was suggested by Ulam and solved in the affirmative by Hyers [15]. ${ }^{1}$ He showed $k$ could be taken as 1 and designated $T$ as an " $\epsilon$ linear transformation." A direct generalization of this result can be obtained by replacing $\epsilon$ by $\psi(x, y)$, a positive monotone nondecreasing symmetric function of $\|x\|$ and $\|y\|$ such that with $\left(\phi(x)=\psi(x, x), \sum_{i=1}^{\infty}\left(\phi\left(2^{i} x\right) / 2^{i}\right)=\mu(x)<\infty\right.$. One can then show by analysis similar to that of Hyers that (4) is valid with $k \epsilon$ replaced by $\mu(x)$. On the other hand (3) and (4) cannot be replaced by

$$
\begin{aligned}
& \bar{L}_{\|x\|,\|y\| \rightarrow \infty}\|T(x+y)-T x-T y\| /(\|x\|+\|y\|)=0, \\
& \bar{L}_{\|x\| \rightarrow \infty}\|T x-U x\| /\|x\|=0 .
\end{aligned}
$$

Indeed with $E$ the real line and $[|x|]$ the integer part of $|x|$ the choice $T x=x\left(1+\sum_{j=1}^{j=\left[\log _{2} \mid x\right]} 1 / j\right)$ or $x$, according as $|x| \geqq 4$ or $|x|<4$, is consistent with $\left(3^{\prime}\right)$. Yet $\left(4^{\prime}\right)$ cannot be satisfied. Obvious trifling modification yields a continuous $T(x)$ of this type.

Suppose now that $X$ and $Y$ refer to the same Banach space in its norm topology, that $S$ is the identity operator, that $T \theta=\theta$, that $\mathfrak{M}$ is again an $\epsilon$ vicinity, and $O$ and $\oplus$ are the vector difference operations. Then (1) is

$$
|\|T x-T y\|-\|x-y\||<\epsilon .
$$

The standard transformation $U$ is now an isometry. For convenience we refer to $T$ as an $\epsilon$ isometry. The first result here was for $X$ a separable Hilbert space. Again $U$ exists satisfying (4) with $k=10$ provided $T$ is an onto transformation. If this last proviso is not met, it is easy to give counter examples to show no $k$ is adequate in (4). Another result in this direction was for the case of $X=C(S)$, the Banach space of continuous functions over a compactum, $S$, where $T$ was required to be single-valued and continuous. Both results are due to Hyers and Ulam $[16,17]$. I was able to treat the $L_{p}(0,1)$

${ }^{1}$ Numbers in brackets refer to the bibliography at the end of the paper. 
case for $p>1$ [4]. Moreover, I was able to demonstrate that a $k$ existed for a uniformly convex space subject to two further conditions. Introduce the following notation: $\delta(\gamma)$ is the supremum of positive numbers compatible with $\left\|z_{1}+z_{2}\right\| \leqq 2(1-\delta(\gamma)) \max \left(\left\|z_{1}\right\|\right.$, $\left.\left\|z_{2}\right\|\right)$ when $\left\|z_{1}-z_{2}\right\| \geqq \gamma \max \left(\left\|z_{1}\right\|,\left\|z_{2}\right\|\right)$ and $\psi(\delta)=\sup \{\gamma \mid \delta(\gamma)$ $\leqq \inf (1, \delta)\}$. The conditions are

$$
\begin{gathered}
\sum \psi\left(2^{-n} C\right)<\infty, \\
L_{\lambda \rightarrow 0,\|z\| \rightarrow 0}\left(\left(\left\|y_{0}+z-\lambda y_{1}\right\|-\left\|y_{0}+z\right\|\right) / \lambda\right)=0,
\end{gathered}
$$

for $\left\|y_{0}\right\|=1$ and $f_{0}\left(y_{0}\right)=1$ where $\left\|f_{0}\right\|=1$ and $z$ and $y$ are annihilated by $f_{0}$. We may remark that the $\epsilon$ isometry $T$ can be shown to be a $36 \epsilon$ linear transformation and that $U$ is the unique distributive operation satisfying $\bar{L}_{\|x\| \rightarrow \infty}(\|T(x)-U(x)\| /\|x\|)<\infty$. The consequences of a condition resembling (7) at least in the case $z=0$ have been recently investigated by Fortet [13]. In the case of $C(S)$ I was able to generalize the Hyers-Ulam result by demonstrating a similar theorem in which $S$ was completely regular instead of a compactum and in which $T$ was neither single-valued nor continuous [5]. No serious effort has been made in the cases successfully treated to determine the "best" value of $k$.

There are two parts to the problem. The first is the determination of $U$; the second, the verification of (4). In all cases studied $U(x)$ turns out to be $L_{n \rightarrow \infty} T\left(2^{n} x\right) / 2^{n}$. In the first cases mentioned the existence of the limit is easy to establish; for instance, (6) guarantees this. The more difficult part is here the proof of (4), and relations of the form of (7) play a role. In $C(S)$ the order of the proof is reversed, and $U(x)$ is derived from the consideration of the known general form of an isometry on such a space in terms of a homeomorphism of $S$ and an analysis of the $T$ maps of certain classes of elements reminiscent of maximal ideals. It is only after (4) is established that the limit definition of $U$ is verified. No general method has emerged as yet, and it is not known whether $k$ exists for every Banach space. Indeed the problem is still open in cases such as $l_{1}$ where the form of an isometry is known.

If now $X$ or $Y$ or both are proper subsets of a Banach space, little is known. If, for instance, $X$ is a subset of the Euclidean plane $Y$, and if we drop the restriction $T(X) \subset X$ even for compact $X$, then no $k$ satisfying (4) need exist. I owe the counter example to Swain [35]. Take for $X$ the triangle with vertices $(-1,0),(1,0)$, and $(0, a)$. We define $T$ by $T(x, y)=(x, y+a(|x|-1))$. Then for each value of $k$ the choice $a=1 / 4 k$ leads to $\epsilon \leqq 2 a^{2}$ and may be shown to give an $\epsilon$ isometry of $X$ and $T(X)$ for which (4) cannot be satisfied for any 
isometry $U$. (Swain has shown that it is true, however, that for a given $k$ one can assign an $\epsilon$ so that (4) and (5) can be satisfied when $X$ is a compactum in the plane.) Actually, however, the more natural problem is that of $X=Y$ where $X$ is a compact subset of the plane or more generally of a Euclidean space. I believe that for each such $X$, $k$ does exist; but I have no proof. If compactness is replaced by metric conditional compactness, there is the possible complication that an isometry may not be onto unless say a residue of some order vanishes [27]; and there seem to be no clear indications regarding the existence of $k$. The fact that compactness may be crucial is shown by the following example. Consider the set $X$ of vertical segments in the plane $\left\{Y^{(n)}\left|Y^{(n)}=\left(x^{n}, y\right), 0 \leqq\right| y \mid \leqq h^{n}\right\}$. Then if $x^{n} \rightarrow \bar{x}, h^{n} \rightarrow 0$, there is a finite $k$; but one can determine a divergent sequence $\left\{x^{n}\right\}$ with $h^{n} \rightarrow 0$ for which no finite $k$ is available.

2. Isometries in groups. When the underlying spaces have algebraic structure, one may ask whether the metric specification of a standard transformation, the isometry, implies algebraic properties. A well known result of this character is the Mazur-Ulam theorem [28] that an isometry of a Banach space which preserves the origin is a linear transformation. It is natural to consider then an isometry of a metric group with, say, left invariant metric which leaves the identity fixed. I point out here that the fundamental difficulties are in the non-Abelian case. The nub of the demonstration of the MazurUlam theorem is the midpoint construction. This has general application and is nontrivial only when the unit sphere is flat. (Thus, the restriction to strictly convex spaces may be waived in some of Rothe's work [32] as observed to me by Charnes.) Aronszajn [2] has given an extension, but this again is for the Abelian situation alone. If one requires that every element be a square, then under weak subsidiary hypotheses the isometry $U$ is a semi-automorphism, that is to say, $U s t s=U s U t U s$. In general this is all one can expect. For the case of a field Hua [19] has shown that $U$ is either a homomorphism or antihomomorphism, and Nakayama [29] has extended this to primary rings with a weak chain condition; but the proofs lean heavily on the existence of two operations. Of course, in the group case (or general ring case), for instance when the groups are product groups, the situation for $T$ can be quite involved. It is not clear how to formulate non-artificial conditions that would guarantee our semiautomorphism is really an automorphism.

Eilenberg [12] showed that any member $T$ of a compact group $G$ of homeomorphisms of a metric space $S$ is an isometry in some equivalent metric associated with the uniform topology. Is the conclusion 
still valid if the compactness of $G$ is dropped and the other hypotheses are strengthened to read $T$ is a topological automorphism of the compact metric group $S$ ? The answer is in the negative as shown by the following counter example I have recently constructed to settle this and other questions. We topologize the additive group of the reals as a compact group $G=\{x\}$, following Halmos [14], by considering it as the character group of the additive group of the rationals $R=\{r\}$ taken with discrete topology. Thus $x$ is considered as $x(r)$, an element of $P^{R}$, where $P$ is the set of reals mod 1 with the customary topology. The topology of $G$ is then indicated by the compact open topology for $P^{R}$. Since the neighborhood basis in $P$ at $p=0$ is denumerable and compact sets in $R$ are finite, it follows that the neighborhood basis in $P^{R}$ satisfies the first denumerability condition. Accordingly, since $G$ is compact, it is metrizable. A sub-basis at $x=0$ is given by the neighborhoods $U(\epsilon, r)=\{x|| r x \mid<\epsilon \bmod 1$, $r \in R\}$. Consider the automorphism $A: x \rightarrow 10 x$. This is plainly 1-1. To show $A$ is topological, it is here sufficient to demonstrate continuity at $x=0$. However, this follows from the observation that for small $\epsilon$

$A: U(\epsilon / 10, r)=\{x \mid r x=10 N \pm \delta, \delta<\epsilon, N=0, \pm 1, \cdots\} \subset U(\epsilon, r)$. If $A$ is an isometry, then $d(0, x)=d(0,10 x)=d\left(0,10^{n} x\right)$, that is, $\left\{10^{n} x \mid n=0, \pm 1, \pm 2, \cdots\right\}$ is included in any metric sphere containing $x$. On the other hand consider $\mathfrak{R}=U(1 / 100,1)$. For $x \neq 0$, we may as well assume that the first nonzero digit follows the decimal. Then either $x$ or $10^{-1} x \in \mathfrak{R}$; that is to say, $\mathfrak{R}$ cannot contain $\left\{10^{n} x\right\}$, and so $A$ is not an isometry.

3. Approximate convexity. A slight generalization of (1) involving dependence of $O$ and $\oplus$ on a parameter is enough to comprehend the case of an affine or convex function on a convex subset of Euclidean $n$ space. The results here were communicated to me by Hyers and Ulam [18]. An $\epsilon$ convex function is real-valued and satisfies

$$
T(h x+(1-h) y)-(h T(x)+(1-h) T(y)) \leqq \epsilon \text { for } 0 \leqq h \leqq 1 .
$$

Here $U$ is convex; and the main theorem asserts that if $X$ is a convex body or merely a convex subset in $Y$, the Euclidean $n$-dimensional space, and if $T$ is continuous, then (4) can be satisfied with $k=n(n+3) / 4(n+1)$.

4. Multiplicative transformations. In view of the cardinal importance in Banach algebras of maximal ideals which are interpreted as 
linear multiplicative functionals, it seems worthwhile to consider purely multiplicative functionals and transformations, as standard transformations, in some detail. Specifically, suppose $X=C(S), S$ compact, and let $Y$ be the real line, with $O$ and $\oplus$ the obvious multiplications. We are interested in $U x y=U x U y$, where $U$ is continuous in the norm topology of $C(S)$. I have recently shown [6] that $U$ is completely determined by the values of $x(s)$ on a denumerable closed set $D$ in $S$ which is independent of choice of $x$. This closed set, which is easily shown to be a compactum in the relative topology, is referred to as a determining set. Some immediate consequences have been noted in unpublished work by Charnes and me [9]. For instance, similar results are valid when $Y$ is the complex field or when $S$ is completely regular and $\beta(S)-S$ is the union of closed $G_{\delta}$ sets where $\beta(S)$ is the Cech compactification of $S$. Furthermore, we have been able to obtain the essential facts about the representation of continuous multiplicative transformations on $C\left(S_{1}\right)$ to $C\left(S_{2}\right)$ for $S_{1}$ and $S_{2}$ compact. For instance, if one requires the transformation to be completely continuous, then a denumerable compactum $D$ in $S_{1}$ is again the determining set in the sense that $(U x)(t)=\prod_{s_{i} \in D}\left|x\left(s_{i}\right)\right|^{\mu_{i}(t)}$. If the transformation is merely continuous, then the correspondence $t \rightarrow D(t)$ is upper semi-continuous. Although $\sum \mu_{i}(t)$ must be continuous, it is not true the individual $\mu_{i}(t)$ 's need be. In this range of ideas there is an expected analogue of the Stone-Banach theorem. Indeed, we have shown that multiplicative isomorphism of the continuous multiplicative functionals on two compacta implies homeomorphism of the compacta.

Cardinal number aspects of multiplicative linear functionals seem in the spirit of the considerations above. For instance suppose $S$ is completely regular and the minimal power of a neighborhood basis at every point is the same cardinal $\alpha$. Let $\Im$ be a free ideal, in Hewitt's terminology, of $C_{B}(S)$, the Banach algebra of bounded continuous functions. Suppose the power of $\{\lambda \mid \Lambda\}$ is $\beta, \beta \leqq \alpha$, and $\left\{x^{\lambda} \mid \Lambda\right\} \subset \Im$. Then for some fixed collection $\left\{s^{\omega} \mid \omega \in \Omega\right.$ a directed set $\}$ $C S$ of power less than or equal to $\mathfrak{B}$, we can assert $L_{\omega} x^{\lambda}\left(s^{\omega}\right)=0$ for each $\lambda$. An interesting consequence is that the free ideal $\Im$ cannot be maximal if it admits a basis of power $\leqq \alpha$.

Other directions of generalization I have in mind are to subrings of $C(S)$ or to the case of $S$ no longer restricted to be compact and to function rings other than $C(S)$. For instance suppose $G$ is a compact Abelian group and $G^{\prime}$ is its discrete character group; then $L_{2}(G)$ is a ring over the complex field $K$ when multiplication is interpreted as convolution while $L_{2}\left(G^{\prime}\right)$ is a ring under pointwise multiplication. 
The intervention of the Fourier transform yields the continuous multiplicative functionals on $L_{2}(G)$ in terms of those on $L_{2}\left(G^{\prime}\right)$. D is finite here. Multiplicative functionals yield the one-dimensional case of representations for the semi group parts of Banach algebras.

The bordering transformations now are what we may term approximately multiplicative. It must be confessed that, in order to preserve interesting properties, the approximately multiplicative transformations turn out to be either disguises of truly multiplicative ones or at best to satisfy somewhat artificial restrictions. For instance, we have with $\Delta x y=\|T x y-T x T y\|,|x|_{i}=\inf _{s \in s_{i}}|x(s)|$, the following alternative definitions:

$$
\begin{aligned}
& \Delta x y \leqq \delta \max \left(\|x\||y|_{i},\|y\||x|_{i}\right) . \\
& \Delta x y \leqq \delta \min \left(\|x\||y|_{i},\|y\||x|_{i}\right) . \\
& \Delta x y \leqq \delta|x|_{i}|y|_{i} . \\
& \Delta x y=0 \text { for }|x y|_{i}=0 . \\
& \Delta x y \leqq \delta|x y|_{i}+\epsilon .
\end{aligned}
$$

For more than one point in $S_{2},(\mathrm{C})$ implies multiplicativity. An example of (A) is $T x=x+|x|$. As an example of (A) and (B) we have $T(x)=|x|_{i}(\operatorname{sign} x(s))\left(\beta(x(s) /\|x\|)^{2}-\alpha\right)$ with $0<\alpha<\beta<2 \alpha$ and $2 \alpha+4 \alpha^{2}<\delta$. This is on $C(S)$ onto $C(S)$.

The multiplicative transformations or approximately multiplicative transformations yield analogues of the Stone-Banach theorem $[3,34]$. I have given a rather general condition of the validity of such a theorem [5]. Indeed, let $S_{i}, i=1,2$, be compact, and refer to $\left\{x|| x\left(s_{0}\right) \mid \leqq \mu\right\}$ as a $\mu$ ideal. Then if there is a map of $C\left(S_{1}\right)$ onto $C\left(S_{2}\right)$ under which $\mu$ ideals in $C\left(S_{1}\right)$ go into subsets of $\mu+\rho$ ideals in $C\left(S_{2}\right)$ and conversely, $S_{1}$ and $S_{2}$ are homeomorphic. We can state, for instance, if $T$ is $1-1$ on $C\left(S_{1}\right)$ onto $C\left(S_{2}\right)$ and satisfies (C) and so does $T^{-1}$, and if $\left(\inf _{s} \sup _{\|X\|=1}(T x)(s)\right)\left(\inf _{t} \sup \|X\|=1\left(T^{-1} X\right)(t)\right)>0$, then $S_{1}$ is homeomorphic to $S_{2}$.

In view of this last theorem and my theorem on the existence of a determining set, we can state: If $T$ satisfies (D) and is 1-1 on $C\left(S_{1}\right)$ onto $C\left(S_{2}\right)$, then $S_{1}$ and $S_{2}$ are homeomorphic.

5. Banach isomorphisms. We now turn attention to some phases of the problem of the Banach isomorphism of two Banach spaces $E_{1}$ and $E_{2}$ [3]. There are interesting modern connotations because the isomorphism may be construed as merely a change of the multiplication definition in a Banach algebra in many cases. The general reference for the results in this section is [9]. Let $\sim$ indicate Banach 
isomorphism. Evidently a multiplication can be introduced in the Banach space $E$ under which it is algebraically isomorphic to $C(M)$, $M$ compact, if and only if $E \sim C(M)$. That $C(M)$ represents either the Banach space or the Banach algebra will cause no confusion. If $M$ and $N$ are compact but not homeomorphic and $C(M) \sim C(N)$, then the multiplication in $C(N)$ can be used to define a new multiplication in $C(M)$. Since the " $C$ norm" is the minimum one among all $B$ isomorphs of $C(M)$ for which the identity of $C(M)$ has norm 1, it may be shown that if two multiplications exist such that $E \sim C(M)$ and $E \sim C(N)$, then $N$ and $M$ are homeomorphic if and only if $\bar{M}=\bar{N}$ where the bar denotes the $w^{*}\left(E^{*}\right)$ closure of the convex hull (when $M$ and $N$ are considered subsets of $E^{*}$ ). It is natural to ask when $(\alpha): C(S) \sim C(S) \times C(S), S$ compact. A set of sufficient conditions is: $S$ contains $A, B$, and $S^{\prime}=A \cup B$, all homeomorphic to it, $S^{\prime}$ satisfies $(\alpha)$, and $A \cap B$ is a retract of $S^{\prime}$. The proof depends on the observation that if $L$ is a retract of the compact set $S$ and if $C_{L}(S)$ $=\{x|x| L=0\}$, then $C(S) \sim C(L) \times C_{L}(S)$. The existence of proper subsets homeomorphic to $S$ is not essential. Indeed $(\alpha)$ is true when $S$ is the unit circle $H$ and in fact $C(H) \sim C(I)$ where $I$ is the unit interval $0 \leqq s \leqq 1$. We have no example in which $C(S)$ is not finitedimensional and yet $(\alpha)$ is not satisfied. Among other results we have shown that if $A_{i}, i=1,2$, is compact and connected and convergent sequences $s_{i}, s_{i}=\left\{a_{i}^{n}\right\}, i=1,2$, exist in $A_{1}$ and $A_{2}$ respectively, and if $B$ is the space $A_{1} \cup A_{2}$ with $a_{1}^{n}$ and $a_{2}^{n}$ identified, then $C(B) \sim C\left(A_{1}\right)$ $\times C\left(A_{2}\right)$. I can make a small remark on the open problem of whether $C(I \times I)$ is isomorphic to $C(I)$, namely, that $C(I \times s) \sim C(I)$ where $s=\{1 / n\} \cup 0$.

6. Orthogonal sequences. We now turn to another aspect of isometric transformations and consider a problem that seems equivalent to the characterization of a class of unitary transformations. In the early 1930's in several lectures and conversations I raised the question as to whether for even or odd functions

$$
\frac{1}{\pi} \int_{-\pi}^{\pi} f(n x) f(x m) d x=\delta_{n m}, \quad n, m=1,2,3, \cdots,
$$

was sufficient to guarantee periodicity of $f(x)$ even under restrictions to class $C$ or $C^{\prime}$ functions, or the like. This question is still open (though I expect an answer in the negative), but more success has been achieved with the characterization of periodic odd functions satisfying (I). C. Mendel and I [8] demonstrated the existence of various types of solutions of (I) under the inessential restriction 
that $f(x)$ is odd. In a subsequent paper [7] I established some necessary and sufficient conditions besides the obvious one that

$$
\sum_{n=1}^{\infty} a_{n} a_{m / n}=\delta_{n m}
$$

where $a_{i}=0$ for nonintegral $i$ and, for $i$ a positive integer, is the $i$ th coefficient in the Fourier sine series expansion of the odd function $f(x)$.

I call attention to merely one facet of the general problem, namely, the fact that if $a_{n}$ vanishes except when $n=p_{1}^{j_{1}} \cdots p_{N}^{j_{N}}$ for fixed primes $p_{1}, \cdots, p_{N}$, the most general solution is obtained by finding the analytic functions of $N$ complex variables which take on boundary values of unit modulus almost everywhere on the distinguished surface $|w|_{i}=1, i=1, \cdots, N$. Call this problem (III). Indeed, if a solution is $\sum c_{i_{1}} \ldots i_{N} w_{1}^{i_{1}} \cdots w_{N}^{i_{N}}$, then the constants $c_{i_{1} \cdots i_{N}}=a_{j}$, $j=p_{1}^{i_{1}} \cdots p_{N}^{i_{N}}$, satisfy (II), and conversely. In the case $N=1$ the function theoretic problem admits the representation $B(w) \exp H(w)$ where $B(w)$ is a Blaschke product and $H(w)$ is the well known Herglotz factor [33]. For $N>1$ no such representation theorem is known, though I have shown, for instance, that if $\left\{c_{i_{1} \cdots i_{n}}\right\} \in l_{1}$, then the representative function must be a special type of rational function [7]. The converse would be the following: if $\left\{a_{n}\right\} \in l_{1}$, then there is a corresponding rational function of a finite number of complex variables satisfying (III); but I have no proof. A solution of (I) and (II) yields a solution of (III). For instance, there is a solution of (I) in terms of the properties of the Dirichlet expansion of a determining function $\phi(z)$. Another criterion is one involving equivalence with the unitary transformation in $L^{2}(0, \infty), g(\lambda)=d \int_{0}^{\infty} H(l \lambda) f(l) l^{-1} d l / d \lambda$, where $H$ is a self-reciprocal kernel defined by $H(\lambda)=\sum_{n>1 / \lambda} a_{n} n^{-1 / 2}$. Indeed, if

$$
\int_{0}^{\infty} \frac{H(l \lambda) H(k \lambda)}{\lambda^{2}} d \lambda=\min (k, 1)
$$

and $\left\{a_{n}\right\} \in l_{1}$, or satisfies even weaker convergence conditions, we have a solution of (I). I believe that if (IV) is satisfied, then $\left\{a_{n}\right\} \in l_{2}$ is sufficient to yield the general solution of (I). If this were established, we should have another tie-up with problem (III). On the other hand, in the general case it is clear we should get the generalization of (III) to holomorphic functions of an infinite number of complex variables.

7. Fixed point index. From now on the bond of association of standard and bordering transformation is the notion of homotopy. 
It is commonplace in the modern literature of analysis and applied mathematics to base existence statements on the Leray-Schauder theorem [26]. This theorem is concerned with a homology type of invariant, namely, the index. Such an index can be defined in the case of a polyhedron in $E_{n}$ even though no restriction on homogeneity in the dimension sense be imposed. Thus let a regular point of the polyhedron be one with a neighborhood in the complex homeomorphic to $E_{s}$ for some $s \leqq n$. A regular fixed point is then an isolated fixed point which is regular, and we assign it the dimension factor $(-1)^{s}$. (Non-regular fixed points are handled by approximating $T$ by $T_{n}$ with regular fixed points.) The index of such a fixed point $x_{0}$ is its order $j$ under the transformation $T$ with respect to the continuous cycle obtained by mapping some sufficiently small $s$ sphere with $x_{0}$ as center by $T$. The index of an open set is then the algebraic sum of the indices of all fixed points contained in the open set under the proviso that none are on the boundary, that is to say, $\sum_{\alpha s}(-1)^{s} j_{\alpha s}$. When the open set is replaced by the polyhedron, this is simply the Lefschetz number.

We now signalize another formulation. The transformation $T$ induces chain and cochain mappings, and Lefschetz has used these to define his now classical zero cycle termed the combinatorial graph of $T$. The Lefschetz number can be considered an intersection number or Kronecker index associated with the graphs of $T$ and of the identity transformation [21]. It would appear that the index is bound to the concept of the Lefschetz number, and it seems likely it should be capable of definition as a Lefschetz number if relative chains and cochains are introduced. Part of the difficulty involved in a definition of the index in this way attaches to the point set assignments of carriers or supports, for there do not exist altogether satisfactory formulations of the notion of general position. Improvement and extension of the intersection theory for the relative homology case would permit corresponding extensions in the effective definition of the index.

The Leray-Schauder degree is an extension of the index beyond the finite complex or Euclidean space stage for the special situation that the transformation is "equivalent" to one restricted to a finite-dimensional linear subspace. The modern method of generalizing topological concepts from polyhedra to spaces is to replace the space and its mappings by a collection of nerves of coverings and to define the relevant notions by suitable limits. This suggests the following schematic plan for generalizing the index of an open set. Let $\mathfrak{N}^{\beta}$ be 
the nerve of the finite open cover $U^{\beta}$, and let $m^{\beta}$ and $n^{\beta}$ refer to the maps of $X$ into $\mathfrak{N}^{\beta}$ and $\mathfrak{N}^{\beta}$ into $X$ respectively. The maps are to be defined so as to guarantee continuity, and this condition can be met for, say, coverings admitting locally finite refinements as shown by Lefschetz [22] and Dowker [11]. We can then associate with $T$ the map $T^{\beta}$ on $\mathfrak{N}^{\beta} \rightarrow \mathfrak{N}^{\beta}$ such that $T^{\beta}=m^{\beta} T n^{\beta}$. The open set $O \subset X$ corresponds to $\left(n^{\beta}\right)^{-1} O$. Suppose $i_{\beta}$ can be defined on $\mathfrak{N}^{\beta}$ for the subcomplex $\left(n^{\beta}\right)^{-1} O$. It is then plausible to define the index for $O$ by the requirement that $i_{\beta}$ remain constant for all $\beta>\beta_{0}$ so that $i_{\beta}$ is in a sense determined by the complex $\mathfrak{N}^{\beta_{1}}, \beta_{1}>\beta_{0}$. Thus, for example, a subclass of the compact spaces with homology groups those of a finitedimensional complex forms a natural category. Both Leray $[23,24$, 25] and later Browder [10] have defined an index for some spaces of this type. Browder's definition is essentially that given above for a compact absolute neighborhood retract which is uniformly locally equiconnected and is referred to below as a $Q$ space. Leray treats a space he designates as convexoide, defined as one admitting a certain class of closed coverings, but no equivalent definition in terms of connectivity properties has been given. Very likely the convexoide spaces form a subclass of the $Q$ spaces.

It is highly desirable that the restriction to compact spaces be waived. For locally compact Hausdorff spaces, I have little doubt that results will obtain of the same sort as in the compact case. However, since the usual infinite-dimensional metric linear spaces are not locally compact in the metric topology, further extension is called for. The schematic program I have given above suggests an extension in which locally finite covers replace finite covers. One must first define the index for locally finite complexes. I may remark that preliminary work indicates the feasibility of such extension of the index to certain paracompact, normal spaces and also extensions of the index in the case of certain general spaces when the transformations are restricted.

8. Fixed point classes. We should expect greater information and more precision by introducing homotopy invariants. The central problem for us here is: Given $T: S \subset S$, what is the minimum number of fixed points of $T$ or, in slightly more general form, what is the minimum number of fixed points of a transformation arising from $T$ by a homotopy? This is the natural question when one has to deal with branches of solutions. In the case of two-dimensional manifolds with or without boundaries, Nielsen [30] introduced the notion of fixed 
point classes. This work was generalized in part by Wecken [36] to the case of polyhedra in Euclidean space and by Browder [10]. Let $\widetilde{S}$ be the universal covering space of a compact, connected, locally connected, and locally simply connected space $S$. This consists of equivalence classes of paths with common initial point, and each class may be indicated by square brackets boxing a representation path. Suppose then $T$ maps $S$ into $S$. Let $P$ denote the path, from the fixed initial point $p_{0}$ to $p$. Let $C$ be a path from $p_{0}$ to $T\left(p_{0}\right)$. We define $\tilde{T}$ on $\tilde{S}$ to $\widetilde{S}$ by $T[P]=[C T(P)]$. Evidently other nonequivalent definitions can be given for $T$ differing in the choice of the paths $C$ from $p_{0}$ to $T(p)$.

Let $\pi\left(S, p_{0}\right)$ be the fundamental group of $S$ based at $p_{0}$ with elements the equivalence classes [ ] of closed loops. Then one shows easily that

$$
\widetilde{T}[\gamma P]=H(\gamma) T[P]
$$

where

$$
H(\gamma)=\left[C T(\gamma) C^{-1}\right] .
$$

A fixed point class is the set of the projections of the fixed points on $\widetilde{S}$ of $[\gamma]^{-1} \tilde{T}[P]$. Under contractibility hypotheses on $S$ one can assure that these fixed points are in the same class if and only if for some joining curve, $\Gamma,(T(p), p) \subset \mathfrak{R}$ for all $p$ on $\Gamma$. The index is assigned to each fixed point class by taking an open set $O$ containing the elements of some class and none on the boundary and using $i(O)$. Fixed point classes with nonzero indices remain fixed point classes of the same index under homotopic variation of $T$. When the index is 0 it is possible that a class may be homotopically correlated with one containing no fixed points.

Consider the homogeneous space derived from $\pi\left(S, p_{0}\right)$ by introducing the equivalence classes or cosets originally suggested by Reidemeister [31], namely, $\alpha \sim \beta$ if and only if for some $[\gamma] \in \pi\left(S, p_{0}\right)$, $H(\gamma) \alpha \gamma^{-1}=\beta$. Denote the coset containing $\alpha$ by $(\alpha)$. Then it was shown by Wecken [36, II] that each fixed point class corresponds to a coset $(\alpha)$. For spaces of the type $(Q)$ it may be shown that the number of fixed point classes is finite. Under homotopy the number of classes with 0 index may change. If the number of cosets is finite, then obviously the number of fixed point classes has a finite upper bound. Otherwise it may be unbounded, though, of course, the number of classes with nonzero index stays fixed.

There are evidently at least as many fixed points as there are fixed 
point classes of nonzero index. Wecken has given an example where there is only one fixed point class, yet no transformation in the homotopy class has less than 2 fixed points.

Again preliminary analysis indicates that compactness may be replaced by $\sigma$ local compactness without seriously affecting the main features of the results. However, further weakening such as, say, to paracompact spaces, runs into difficulties that suggest only restricted homotopies be used in view of Kakutani's result [20] that the Hilbert sphere $\{x \mid\|x\|=1\}$ is homotopic to a point over itself.

I conclude with a few comments about sets of fixed points. In a general way it has been shown by Aronszajn [2] that when a transformation into a compactum can be uniformly approximated by transformations admitting unique solutions, then the fixed point set is an intersection of a decreasing sequence of absolute retracts and is referred to as an $R_{\delta}$ after the usual signification of $\delta$. An $R_{\delta}$ set is like an absolute retract as regards its trivial fundamental and homology groups, but may have different local properties as indicated by the mathematician's vade mecum, the $\sin 1 / x$ curve plus the vertical segment at $x=0$, which obviously is an $R_{\delta}$. I mention in passing that it would be of interest to give an internal characterization of an $R_{\delta}$. The precise statement of Aronszajn's result is: If $T$ is completely continuous on the Banach space $E$ into itself, and if there is a sequence of completely continuous transformations $T_{n}$ such that (a) $\epsilon_{n}>\left\|T x-T_{n} x\right\|$ for $x \in K$ a bounded closed set, with $\epsilon_{n} \downarrow 0$; (b) $w=H_{n} z=z-T_{n} z$ maps $K$ in 1-1 fashion on a set containing a fixed sphere in $E$ independent of $n$ (this guarantees uniqueness in $K$ of solution of $H_{n} z=w$ for $w$ of sufficiently small norm); then the set of fixed points of $T$ is an $R_{\delta}$. There seem obvious applications of the index and fixed point class concepts in extending this result. Moreover, quite apart from the extension where compact replaces compact metric, another direction of generalization would be to the cases that the approximating transformations were not necessarily singlevalued. There is reason to surmise then that the corresponding fixed point set would be an intersection of absolute neighborhood retracts.

\section{BIBLIOGRAPHY}

1. N. Aronszajn, Caractérisation métrique de l'espace de Hilbert, des espaces vectorieles et de certains groupes métriques, C.R. Acad. Sci. Paris vol. 201 (1935) pp. 811813 and $873-875$.

2. - Le correspondant topologique de l'unicité dans la thêorie des équations differrentielles, Ann. of Math. (2) vol. 43 (1942) pp. 730-738.

3. S. Banach, Théorie des opérations linéaires, Warsaw, 1932. 
4. D. G. Bourgin, Approximate isometries, Bull. Amer. Math. Soc. vol. 52 (1946) pp. 704-714.

5. - Approximately isometric and multiplicative transformations on continuous function rings, Duke Math. J. vol. 16 (1949) pp. 385-397.

6. - Multiplicative transformations, Proc. Nat. Acad. Sci. U.S.A. vol. 36 (1950) pp. 564-570.

7. - $A$ class of sequences of functions, Trans. Amer. Math. Soc. vol. 60 (1946) pp. 478-518.

8. D. G. Bourgin and C. Mendel, Orthogonal sets of periodic functions of the type $f(n x)$, Trans. Amer. Math. Soc. vol. 57 (1945) pp. 332-363.

9. D. G. Bourgin and A. Charnes, Unpublished.

10. F. Browder, Thesis, Princeton University.

11. C. H. Dowker, An extension of Alexandroff's mapping theorem, Bull. Amer. Math. Soc. vol. 54 (1948) pp. 386-391.

12. S. Eilenberg, Sur les groupes compacts d'homéomorphie, Fund. Math. vol. 28 (1937) pp. 75-80.

13. R. Fortet, Remarques sur les espaces uniformément convexes, Bull. Soc. Math. France vol. 69 (1941) pp. 23-46.

14. P. Halmos, Comment on the real line, Bull. Amer. Math. Soc. vol. 50 (1944) pp. 877-878.

15. D. H. Hyers, On the stability of the linear functional equation, Proc. Nat. Acad. Sci. U.S.A. vol. 27 (1941) pp. 222-224.

16. D. H. Hyers and S. Ulam, On approximate isometries, Bull. Amer. Math. Soc. vol. 51 (1945) pp. 388-392.

17. - Approximate isometries of the space of continuous functions, Ann. of Math. (2) vol. 48 (1947) pp. 385-389.

18. - Unpublished.

19. L. K. Hua, On the automorphisms of a field, Proc. Nat. Acad. Sci. U.S.A. vol. 35 (1949) pp. 386-389.

20. S. Kakutani, Topological properties of the unit sphere of a Hilbert space, Proc. Imp. Acad. Tokyo vol. 19 (1943) pp. 269-271.

21. S. Lefschetz, Algebraic topology, Amer. Math. Soc. Colloquium Publications, vol. 27, New York, 1942. 1942.

22. - Topics in topology, Annals of Mathematics Studies, no. 10, Princeton,

23. J. Leray, Sur la forme des espaces topologiques et sur les points fixes des représentations, J. Math. Pures Appl. (9) vol. 24 (1945) pp. 95-167.

24. - Sur la position d'un ensemble fermé de points d'un espace topologique, J. Math. Pures Appl. (9) vol. 24 (1945) pp. 169-199.

25. - Sur les équations et les transformations, J. Math. Pures Appl. (9) vol. 24 (1945) pp. 201-248.

26. J. Leray and J. Schauder, Topologie et équations fonctionnelles, Ann. École Norm. (3) vol. 51 (1934) pp. 45-78.

27. A. Lindenbaum, Contributions a l'étude de l'espace métrique I, Fund. Math. vol. 8 (1926) pp. 209-222.

28. S. Mazur and S. Ulam, Sur les transformations isométrique d'espaces vectoriels, normés, C.R. Acad. Sci. Paris vol. 194 (1932) pp. 946-948.

29. T. Nakayama, Unpublished.

30. J. Nielsen, Untersuchungen zur Topologie der geschlossen zweiseitigen Fläche, I, 
Acta. Math. vol. 50 (1927) pp. 189-358; II, vol. 53 (1929) pp. 1-76; III, vol. 58 (1932) pp. 87-167.

31. K. W. Reidemeister, Automorphismen von Homotopiekettenringen, Math. Ann. vol. 112 (1936) pp. 586-593.

32. E. Rothe, The theory of topological order in some linear topological spaces, Iowa State College Journal of Sciences vol. 13 (1939) pp. 373-390.

33. W. Seidel, On the distribution of values of bounded analytic functions, Trans. Amer. Math. Soc. vol. 36 (1934) pp. 201-226.

34. M. H. Stone, Applications of the theory of Boolean rings to general topology, Trans. Amer. Math. Soc. vol. 41 (1937) pp. 375-481.

35. R. L. Swain, Unpublished.

36. F. Wecken, Fixpunktklassen, I, Math. Ann. vol. 117 (1939) pp. 659-671; II, vol. 118 (1941-1943) pp. 216-234; III, vol. 118 (1941-1943) pp. 544-577.

UNIVERSITY OF ILLINOIS 